\title{
Vorwort und Dank
}

Das vorliegende Buch ist eine leicht überarbeitete Fassung meiner Habilitationsschrift, die ich im Herbstsemester 2017 an der Universität Bern eingereicht habe und auf deren Grundlage mir Anfang 2018 die Venia docendi für Kunstgeschichte erteilt worden ist.

Was als eine Studie über das Material Gips im Werk der Bildhauer Antonio Canova, Bertel Thorvaldsen, Vincenzo Vela und Auguste Rodin begonnen hatte, resultierte in einer künstlermonografischen Untersuchung zu Thorvaldsens öffentlicher Persona. Entscheidend dabei war mein erster Besuch in dessen Museum in Kopenhagen im Sommer 2013. Als ultimative Visualisierung des beispiellosen Künstlerkults um Thorvaldsen nimmt sein Museum auch in diesem Buch eine zentrale Rolle ein.

Die Entstehung dieser Monografie wurde von zahlreichen Menschen begleitet. Es liegt in der Natur eines Vorwortes, der empfundenen Dankbarkeit gegenüber Weggefährtinnen und Weggefährten, Kolleginnen und Kollegen, Freundinnen und Freunden nur begrenzt Ausdruck verleihen zu können. Deshalb möchte ich an dieser Stelle diejenigen Personen hervorheben, die mir während meiner Arbeit an diesem Buch am längsten oder in besonders intensiven Phasen zur Seite standen.

Der erste Dank gilt Christine Göttler, die meine Forschungen stets mit Interesse und kritischen Nachfragen verfolgt und dadurch wesentlich geprägt hat. Dasselbe gilt für Oskar Bätschmann, Pascal Griener und Johannes Myssok, die die Entstehung dieser Arbeit ebenfalls von Anfang an beobachtet und deren Horizont mit wertvollen Hinweisen erweitert haben. Auch meiner Habilitationskommission, bestehend aus Christine Göttler, Johannes Myssok, Oliver Lubrich, Bernd Nicolai und Stefan Rebenich, bin ich zu Dank verpflichtet.

Der Schweizerische Nationalfonds (SNF) hat meine Arbeit mit einem dreijährigen Stipendium gefördert, das mir Forschungsaufenthalte an Thorvaldsens Museum in Kopenhagen, der University of Michigan in Ann Arbor und am University College London sowie eine Rückkehrphase an die Universität Bern ermöglichte. Die Gespräche mit den Mitarbeitenden an den Gastinstitutionen haben meine Forschungen in unschätzbarem Maß bereichert. Dafür danke ich besonders Ernst Jonas Bencard, Karen Benedicte Busk-Jepsen, Kristine Bøggild Johannsen, Elettra Carbone, Kira Kofoed, Nanna Kronberg Frederiksen, Stig Miss, Lejla Mrgan, Alexander Potts, Laila Skjøthaug und Claire Thomson. 
Ein großer Dank gilt auch dem De Gruyter Verlag, namentlich Katja Richter und Arielle Thürmel, für die angenehme Zusammenarbeit. Bénédicte Savoy, Michael Thimann und Gregor Wedekind danke ich für die Aufnahme des Buches in die Schriftenreihe Ars et Scientia. Die Veröffentlichung wurde ermöglicht durch einen Publikationsbeitrag des SNF.

Michael Guery ( $\dagger$ ) begleitete meine Forschungen zu diesem Buch von den allerersten Ideen bis zur Einreichung als Habilitationsschrift. Auch ihm möchte ich auf diesem Weg meinen Dank aussprechen, auch wenn dieser ihn nicht mehr erreichen wird.

Ein nicht in Worte zu fassender Dank für alles gilt schließlich meinen Eltern.

T. S., im Januar 2021 